\title{
Ultraviolet spectrophotometry of flares on "quiescent" $M$ and K dwarf exoplanet hosts
}

\author{
R. O. Parke Loyd ${ }^{1}$, Kevin France ${ }^{1}$ and Allison Youngblood ${ }^{1}$ \\ ${ }^{1}$ Laboratory for Atmospheric and Space Physics, University of Colorado, 600 UCB, Boulder, \\ CO 80309 \\ email: robert.loyd@colorado.edu
}

\begin{abstract}
We present an analysis of a sample of flares on "quiescent" (i.e. non-flare) M and K stars using temporally resolved UV spectroscopy from the growing body of MUSCLES Treasury Survey data. Specifically, our analysis quantified the response of the far-UV C II, Si III, Si $\mathrm{IV}$, and $\mathrm{N} \mathrm{V}$ emission lines and the far-UV continuum during the flares. Using these tracers, we examined one representative event on GJ 832. In concordance with flares recorded on the Sun and AD Leo, the MUSCLES flares are well fit by a power law relationship of similar slope in frequency versus energy. Flares can strip atmospheric mass from orbiting planets, adversely affecting their long-term habitability. To gauge the amplitude of this effect, we computed an energy-balance upper-limit on the amount of atmosphere a large flare might remove from an orbiting Earth due purely to elevated EUV flux and found this limit to be modest relative to Earth's atmospheric mass.
\end{abstract}

Keywords. low-mass stars, ultraviolet flares, exoplanet atmospheres

\section{Introduction}

Observations of low-mass, magnetically-active stars such as AD Leo have recorded many flares where ultraviolet line emission peaks at a level many times that of quiescence (e.g. Hawley et al. 2003, Loyd \& France 2014). This behavior can be resolved in both wavelength and time for nearby cool stars with HST's UV spectrographs, COS and STIS, tracing energy input, mass motions, and plasma properties of different ionic species through the evolution of UV emission lines.

So far, detailed UV observations of low-mass stellar flares have typically targeted the well-known "flare" stars AD Leo, AU Mic, and EV Lac (Hawley et al. 2003; Robinson, Linsky, Woodgate, \& Timothy 2001; Osten et al. 2005), but the ongoing HST observations for the MUSCLES Treasury Survey (Measurements of the Ultraviolet Spectral Characteristics of Low-mass Exoplanet Systems) are changing this. MUSCLES' primary purpose is compiling a spectral library for 11 nearby, low-mass exoplanet hosts. However, MUSCLES will also augment the existing body of UV flare data with high cadence (sub-minute) UV spectrophotometry of these "quiescent" hosts - stars not noted for high levels of magnetic activity. An overview of the MUSCLES project, description of its spectral data products, and discussion of a UV flare on GJ 832 (for which additional spectral information is presented here) and an x-ray flare on GJ 581 can be found in the conference proceeding of Kevin France (France, Loyd, \& Brown 2015; this volume). Here we present an early analysis of all flares detected on the 8 hosts observed thus far.

\section{Portrait of a Flare on a MUSCLES Star: GJ 832}

GJ 832 exhibited a strong flare at 2014 Oct 10 12:16 UT. Examining the integrated line fluxes as a function of time during this flare, plotted in Figure 1 (left axes), reveals that it is a compilation of three spikes in emission, the highest peaking at some 80 times 

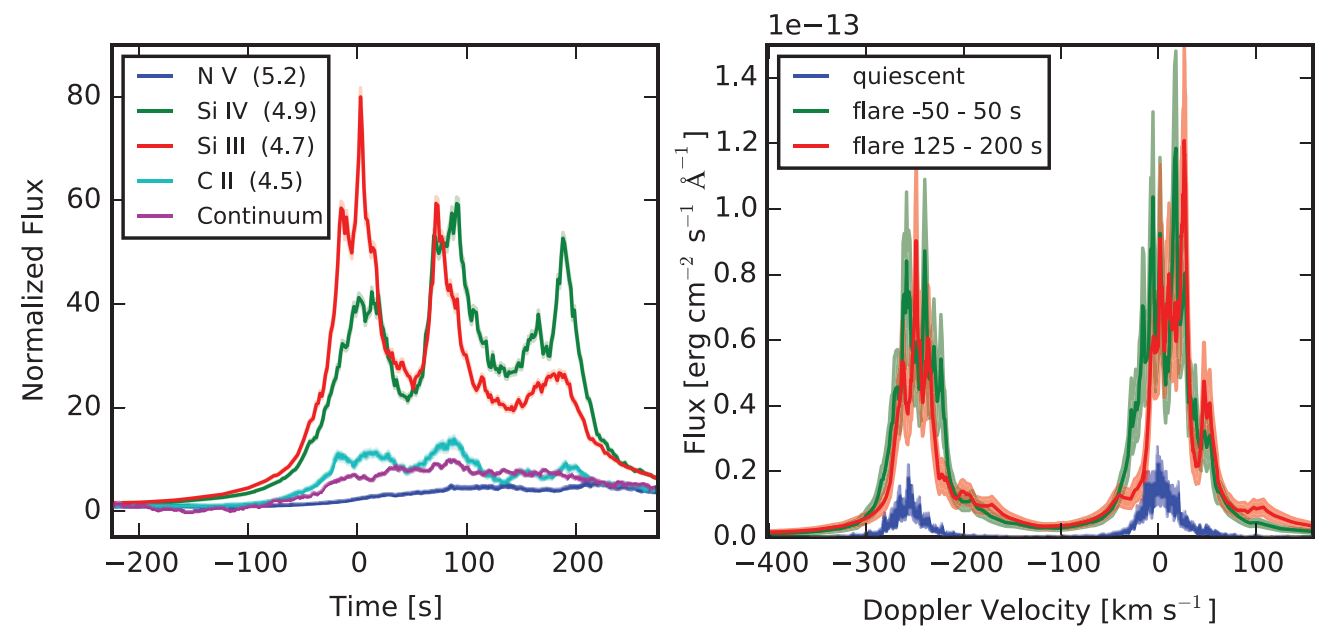

Figure 1. Left: Emission light curves during the GJ 832 flare. The legend shows line formation temperatures in dex (from Dere et al. 2009) in parenthesis. Line wavelengths are C II $\lambda \lambda 1334,1335$, Si III $\lambda 1206$, Si IV $\lambda \lambda 1393,1402$, and N V $\lambda \lambda 1238,1242$. Right: Spectra of the C II lines during three phases of the flare, with times referenced to the light curves. Both plots are adaptively binned for constant $\mathrm{S} / \mathrm{N}$ and oversampled (hence, consecutive points are not statistically independent) to show maximum detail. Uncertainties are represented as translucent areas around each line (too small to be clear in the light curves).

the quiescent level. These three peaks suggest three distinct (though related) reconnection events. The delayed and more gradual response of the N V $\lambda \lambda 1239,1243$ lines and FUV continuum suggest that energy deposition was tightly confined to a region of the atmosphere with high Si III and Si IV populations, afterwards propagating to the hotter (presumably higher altitude) and cooler (presumably lower altitude) regions of $\mathrm{N} \mathrm{V}$ and continuum emission.

Resolving atomic lines at different phases of the flare, as with the C II $\lambda \lambda 1334,1335$ lines plotted in Figure 1 (right axes), shows that bulk mass flows develop after the impulsive event, as expected. The redshifted peaks from downflowing material at 50 and $100 \mathrm{~km} / \mathrm{s}$ are the most prominent. There is also the suggestion of outward flowing material as a blueshifted peak at $-50 \mathrm{~km} / \mathrm{s}$. For context, the stellar escape velocity is roughly $600 \mathrm{~km} / \mathrm{s}$.

\section{MUSCLES Flares in the Context of AD Leo and the Sun}

We compiled energy and peak flux measurements in strong emission lines and continuum for all flares observed in the MUSCLES data as of 2015 Aug 10. Roughly 2/3 of these flares occurred on the M5.0 star GJ 876 and 1/3 on other targets (K2.0 and M1.5-M3.5). Creating energy-frequency distributions of flares from these aggregated measurements in Si III and Si IV emission (Figure 2) shows the expected power-law distribution. The distribution's slope is identical, within errors, to the distribution of C III $\lambda 977$ flares on the Sun and Si IV flares on the well-studied flare star AD Leo. However, flare rates vary substantially. A comparison with solar rates is complicated by the use of different emission lines, but a direct comparison is possible with AD Leo, showing that AD Leo flares more frequently than the MUSCLES stars by a factor of a few.

\section{Implications for Exoplanets}

Flares in the transition-region line emission of the 8/11 targets analyzed for this work occupied $10 \%$ of the observed time and added $30-40 \%$ to the cumulative energy emitted. 


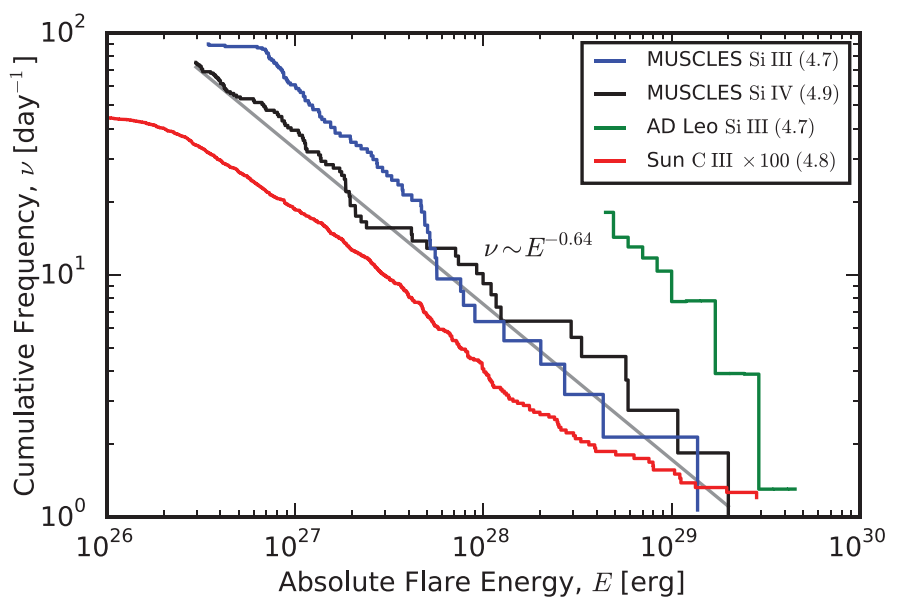

Figure 2. Cumulative distribution of flares as frequency versus absolute energy for all MUSCLES Si III and Si IV flares, solar C III ( $\lambda$ 977) flares observed by SDO EVE (from the catalog of Hock 2012), and AD Leo Si IV flares (from Loyd \& France 2014). Numbers in legend parenthesis are formation temperatures in dex (Dere et al. 2009). The gray line shows a power law fit to the MUSCLES Si IV flares.

These impulsive energy inputs could erode the atmospheres of otherwise habitable planets through both high energy radiation, namely EUV, and ejected particles (Lammer et al. 2007). EUV driven atmospheric escape is constrained by balancing EUV flux with gravitational potential. Assuming that EUV flux scales with Si IV flux during a flare, this balance limits the amount of atmosphere that the GJ 832 flare could liberate from the gravitation well of an Earth-like planet (such as GJ 832's habitable-zone super Earth; Wittenmyer et al. 2014) to below $10^{11} \mathrm{~g}$ - or $10^{-11}$ Earth-atmospheres by mass. Taking this estimate to the extreme by extrapolating the power-law fit in Figure 2 to the energy of a once-per-10 Gy flare, no more than $0.5 \%$ of an Earth-atmosphere could be removed by direct EUV heating from a single event. This suggests that EUV flare radiation may not play a direct role in the erosion of planetary atmospheres. However, impulsive EUV heating will play a potentially important indirect role by inflating atmospheres, thereby rendering them more susceptible to particle erosion (Lammer et al. 2007). Future work will explore the time-dependent response of vertical structure in planetary atmospheres to the impulsive radiation inputs measured by MUSCLES.

\section{References}

Dere, K. P., Landi, E., \& Young, P. R., et al. 2009, A\&A, 498, 915

France, K., Loyd, R., \& Brown, A. 2015, IAU320, 29, 2228599

Hawley, S., Allred, J., Johns-Krull, C., et al. 2003, ApJ, 597, 535

Hock, R. 2012, PhDT

Lammer, H., Lichtenegger, H. I. M., Kulikov, Y. N., et al. 2007, AsBio, 7, 185

Loyd, R. \& France, K. 2014, ApJS, 211, 9

Osten, R., Hawley, S., Allred, J., Johns-Krull, C., \& Roark, C. 2005, ApJ, 621, 398

Robinson, R., Linsky, J., Woodgate, B., \& Timothy, J. 2001, ApJ, 554, 368

Wittenmyer, R., Tuomi, M., Butler, R., et al. 2014, ApJ,791, 114 